\title{
Ab. Initio Study of the Structural, Elastic, Electronic and Optical Properties of $\mathrm{Cu}_{3} \mathrm{~N}$
}

\author{
A. Rahmatia, ${ }^{a, c *}$, M. Ghoohestani ${ }^{\mathrm{d}}$, H. Badehian ${ }^{e}$, M. Baizaee $^{a}$ \\ ${ }^{a}$ Department of Physics, Faculty of Science, Vali-e-Asr University of Rafsanjan, Rafsanjan, Iran \\ ${ }^{b}$ Nano Research Group, Vali-e-Asr University of Rafsanjan, Rafsanjan, Iran \\ 'Thin Film Laboratory, Faculty of Science, Vali-e-Asr University of Rafsanjan, Rafsanjan, Iran \\ ${ }^{d}$ Nano Research Centre, Department of Physics, Malek Astar University of Technology, \\ Shahinshahr, Isfahan, Iran \\ ${ }^{e}$ Condensed Matter Lab, Department of Physics, Faculty of Science, Shahid Chamran University, Ahvaz, Iran
}

Received: February 20, 2013; Revised: January 14, 2014

\begin{abstract}
Electronic, optical, elastic, properties of Copper nitride $\left(\mathrm{Cu}_{3} \mathrm{~N}\right)$ in cubic anti- $\mathrm{ReO}_{3}$ phase have been studied using the full-potential augmented plane waves (FP-LAPW) within density functional theory (DFT) framework. Generalized gradient approximation (GGA), local density approximation (LDA), Perdew- Burke-Ernzerhof generalized parameterization of gradient approximation (GGA-PBE), and new modified Becke and Johnson GGA (MBJ-GGA) have been used for exchange-correlation potentials. The structural properties such as equilibrium lattice parameter, bulk modulus and its pressure derivative have been obtained and optimized. The Hubbard potential has been enhanced to improve bandgap energy. Optical properties, such as the dielectric function, refractive index, extinction index, and optical band gap, were calculated for radiation up to $14 \mathrm{eV}$. The chemical bonding in $\mathrm{Cu}_{3} \mathrm{~N}$ was discussed by three method electronegativity concept, B/G ratio, and charge density distribution. Moreover, Elastic constants, Young's modulus, shear modulus, Poisson's ratio, sound velocities for longitudinal and shear waves, Debye average velocity and Debye temperature have been calculated. The estimated structural, elastic and other parameters are in good agreement with experimental data. The calculation exhibits that $\mathrm{Cu}_{3} \mathrm{~N}$ is a direct semiconductor $(0.7-1.12 \mathrm{eV})$ with ductile and ionic identity.
\end{abstract}

Keywords: band structure, density of states, elastic constants, Debye temperature The PACS: 71.20.Be, 71.20.Gj, 81.05.Bx describe the paper well

\section{Introduction}

Many interests were concentrated on copper nitride for several reasons; copper nitride can be used as an optical engraving medium for optoelectronic devices ${ }^{1}$, a barrier material in spin tunnel junction ${ }^{2}$, a high density optical storage media ${ }^{3,4}$, micrometric conductive dots and lines ${ }^{5}$ and good candidate for hybrid inorganic solar cells $\mathrm{s}^{6}$.

Copper nitride has several phases among which cubic anti- $\mathrm{ReO}_{3}$ structure is more stable. In this phase Copper nitride has simple cubic structure and is crystallized in space group pm-3 m (221) with atomic positions $\mathrm{Cu}(0,0,0)$ and $\mathrm{N}(1 / 2,1 / 2,1 / 2)$. Unit cell of $\mathrm{Cu} 3 \mathrm{~N}$ is illustrated in Figure 1.

There are always noticeable divergences between values of theoretically calculated band gap energy and experimental ones. According to the theoretical works, $\mathrm{Cu}_{3} \mathrm{~N}$ is semiconductor with narrow band gap in 0.23 to $0.9 \mathrm{eV}$ regions ${ }^{7,8}$ whereas, experimental results change between 0.8 to $1.9 \mathrm{eV}^{6,9-11}$.

In this study, we have studied structural, elastic, optical, and electronic properties of yttrium oxide compound in cubic phase using the full-potential augmented plane waves (FPLAPW) within density functional theory (DFT) framework. Moreover, we have used new approximation for exchange and correlation terms (MBJ-GGA) for band calculation

*e-mail: a.rahmati@vru.ac.ir which greatly improves the band gap. Our results agree very well with experimental studies. Some experimental results were incorporated to validate theoretical results.

\section{Computational Method}

The full-potential linear augmented plane wave (FP-LAPW) method implemented in WIEN2K (2011) computer package ${ }^{12}$. In this method the space is divided into an interstitial region (IR) and non-overlapping (MT) spheres centered at the atomic sites. the basis set consists of plane waves, in the IR region. In the MT spheres, the basis sets is described by radial solutions of the one particle Schrodinger equation (at fixed energy) and their energy derivatives multiplied by spherical harmonics. To achieve energy eigenvalues convergence, the wave functions in the interstitial region were expanded in plane waves with a cut-off, $K_{\max }=14 / R_{m t}$ where $R_{m t}$ denotes the smallest atomic sphere radius and $\mathrm{K}_{\max }$ gives the magnitude of the largest $\mathrm{K}$ vector in the plane wave expansion. For $\mathrm{Cu}_{3} \mathrm{~N}$, the radius values $\mathrm{R}_{\mathrm{mt}}$ are taken to be 1.7 and 1.5 atomic units (a.u.) for $\mathrm{Cu}$ and $\mathrm{N}$ respectively. The full-potential methods the calculation is dependent on the choice of sphere radii in the full-potential methods. The valence wave functions inside the spheres are expanded up to $l_{\max }=10$, while the charge 
density was Fourier expanded up to $\mathrm{G}_{\max }=14$ (a.u. $)^{-1}$. The exchange- correlation (XC) effect was treated by the generalized gradient approximation (GGA), GGA+U, mBJ and $\mathrm{mBj}+\mathrm{U}$. The self-consistent calculations are considered to be converged when the total energy of the system is

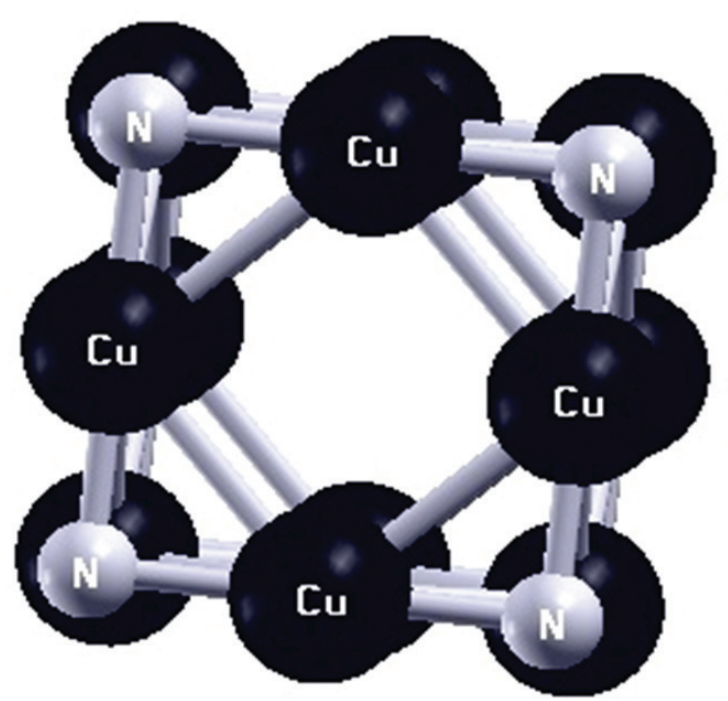

Figure 1. Unit cell of $\mathrm{Cu}_{3} \mathrm{~N}$.

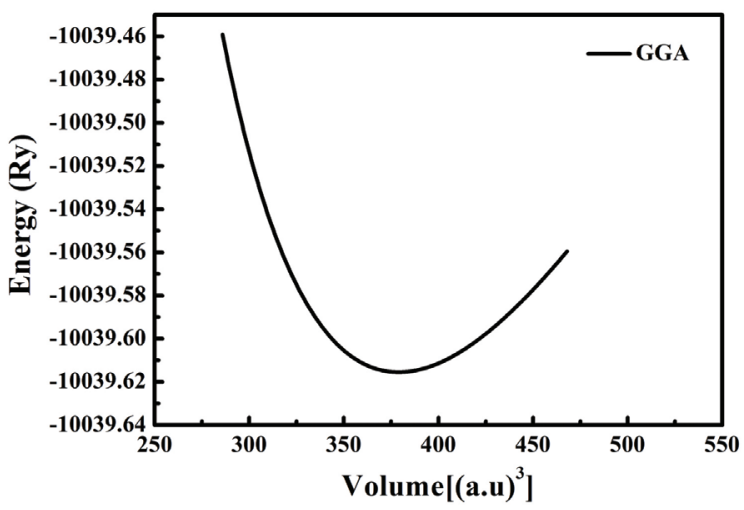

(a) stable within $10^{-4}$ Ry. The integrals over the irreducible Brillouin zone (IBZ) are performed up to $35 \mathrm{k}$-points, using the Monkhorst-Pack special k-points approach ${ }^{13}$. To validate the results, the calculated reflectance spectra, refractive index and absorption coefficient were compared with their corresponding experimental results.

\section{Results and Discussions}

\subsection{Structural properties}

Figure 2 shows the calculated total energy of $\mathrm{Cu}_{3} \mathrm{~N}$ structure by GGA, LDA and GGA+SP approaches. To obtain the ground state properties, the total energies are fitted to Murnaghan ${ }^{14}$ equation of state

$$
E(V)=E_{0}+\frac{B_{0} V_{0}}{B^{\prime}}\left[\frac{V}{V_{0}}+\frac{\left(V / V_{0}\right)^{1-B^{\prime}}-B^{\prime}}{B^{\prime}-1}\right]
$$

where $\mathrm{P}$ is the pressure, $\mathrm{V}$ is the volume at pressure $\mathrm{P}, \mathrm{V}_{0}$ is the volume at ambient pressure, $\mathrm{B}_{0}$ is the bulk modulus at ambient pressure and $\mathrm{B}^{\prime}$ is the pressure derivative of bulk modulus $\mathrm{B}_{0}$.

The equilibrium lattice constant, bulk modulus and pressure derivative of the bulk modulus are given in Table 1, together with some theoretical results and the available experimental data. It is clearly seen that the LDA

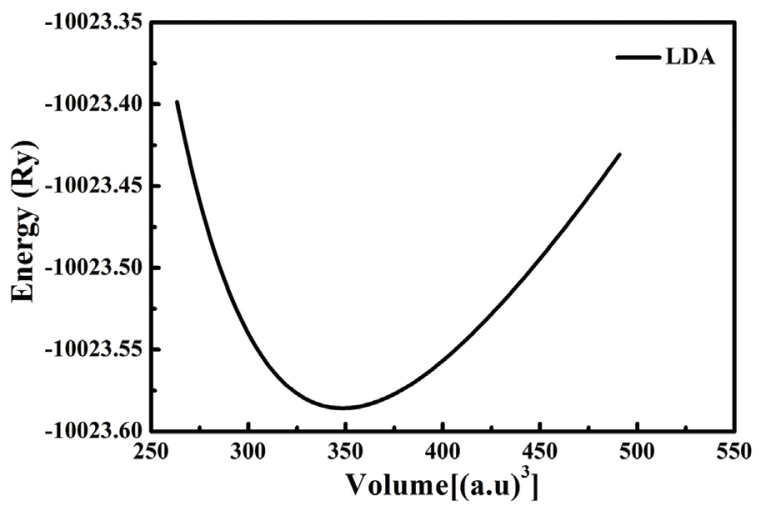

(b)

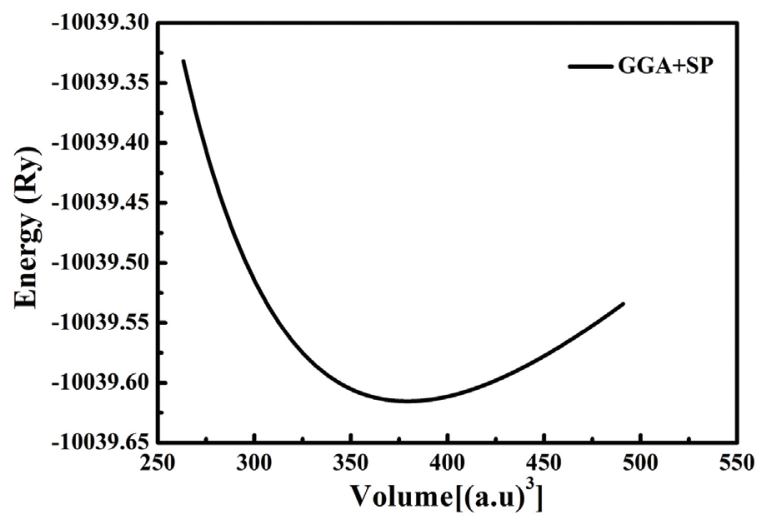

(c)

Figure 2. Total energy versus the atomic volume for cubic $\mathrm{Cu}_{3} \mathrm{~N}$. by (a) GGA, (b) LDA and (c) GGA+SP approximations. 
under-estimates (over-estimates) the lattice parameters (Bulk modulus) while the GGA and GGA+SP results are in reasonable agreement with the experimental and other calculated values.

\subsection{Electronic properties}

In order to investigate electronic properties, the band structure and density of states (DOS) were calculated. Figure 3 depicts the calculated band structure and total DOS for $\mathrm{Cu}_{3} \mathrm{~N}$ by GGA. There are three regions. First; a sharp peak under $-15 \mathrm{eV}$ (related to Nitrogen 2 s orbitals), second; wide valance band about $8 \mathrm{eV}$, third; conduction band. From partial DOS (Figure 4), there is strong hybridization between $\mathrm{N}$ and $\mathrm{Cu}$ states mostly related to $\mathrm{N} 2 \mathrm{p}$ and $\mathrm{Cu} 3 \mathrm{~d}$ orbitals. Moreover, it is understood that $\mathrm{Cu}_{3} \mathrm{~N}$ compound is a semiconductor with indirect band gap. The maximum valance band is at $\mathrm{R}$ point and the minimum conduction band is at $\mathrm{M}$ point.

There are noticeable differences between values of theoretically calculated band gap energy and experimental ones. According to the theoretical works, $\mathrm{Cu}_{3} \mathrm{~N}$ is

Table 1. The equilibrium lattice constant $a(\AA)$, bulk modulus $B_{0}$ (GPa), pressure derivative of bulk modulus $B$ ' calculated using $G G A, L D A, G G A+S P$ approximation.

\begin{tabular}{|c|c|c|c|}
\hline ural & $a(\AA)$ & $B_{0}(\mathbf{G P a})$ & $B^{\prime}$ \\
\hline GGA & 3.829 & 113.9 & 4.50 \\
\hline LDA & 3.72 & 145.98 & 4.87 \\
\hline $\mathrm{GGA}+\mathrm{SP}$ & 3.829 & 113.9 & 4.50 \\
\hline Other results & $3.82-3.85^{1-9}$ & $104-114^{7,9,12}$ & $4.66-5.26^{7,12}$ \\
\hline
\end{tabular}

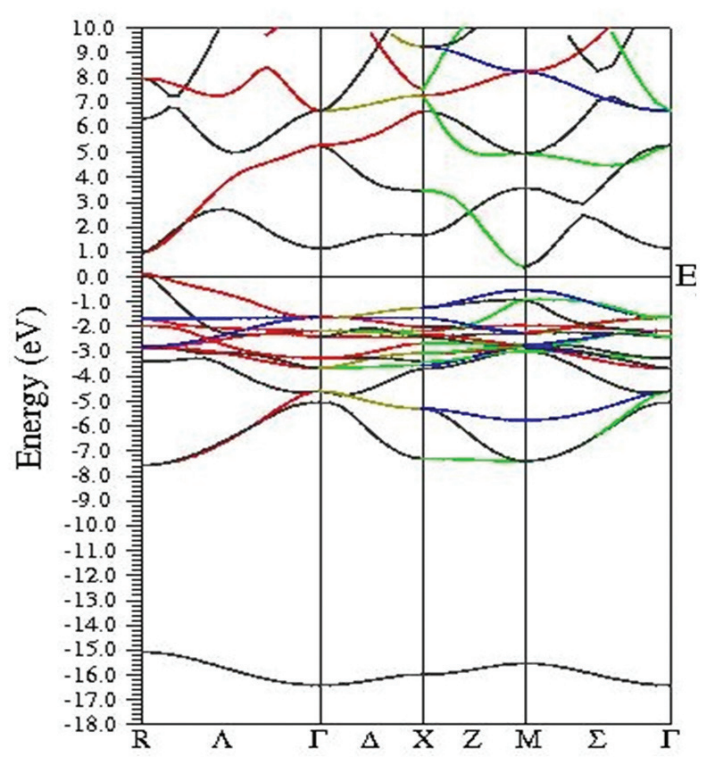

Figure 3. Band structure of $\mathrm{Cu} 3 \mathrm{~N}$ at the ground state by using GGA approximation. semiconductor with narrow band gap in 0.23 to $0.9 \mathrm{eV}$ regions ${ }^{6,7}$ whereas, experimental results change between 0.8 to $1.9 \mathrm{eV}^{[6,9-11]}$. The calculated bandgap energy by different approaches was listed in Table 2. By applying GGA-PBE (PBE0) and MBJ-GGA approaches, the direct band gap were estimated to be 0.85 and $1.04 \mathrm{eV}$ that improved in comparison to GGA and predicted in the range of experimental results.

To coincide the calculated bandgap energy with the experimental one, the Hubbard parameter $U$ was introduced to guarantee spatial distribution of the partially filled $\mathrm{d}$ orbitals ${ }^{15}$. The $\mathrm{U}$ values were calculated to be $5.2 \mathrm{eV}$ for $\mathrm{Cu}_{3} \mathrm{~N}$ using Wien $2 \mathrm{~K}$ package. By applying $\mathrm{GGA}+\mathrm{U}$ and $\mathrm{MBJ}+\mathrm{U}$ approaches, the band gap was calculated 0.95 and $1.12 \mathrm{eV}$, respectively. This confirms the latter approach was suitable to calculate band gap in semiconductors as reported by Tran et al. ${ }^{16}$. Using MBJ, the band gap was obtained $1.04 \mathrm{eV}$ that improve our previous results and other's results. Then effective potential (U) was added to MBJ and the band gap is calculated $1.12 \mathrm{eV}$ (see Table 2). In Figure 4, the band structure, the total and atomic densities of states of $\mathrm{Cu}_{3} \mathrm{~N}$ by $\mathrm{GGA}+\mathrm{U}$ and $\mathrm{MBJ}+\mathrm{U}$ are depicted to analogy with other approaches.

\subsection{Optical properties}

The optical properties of matter can be described by the complex dielectric function $\varepsilon(\omega)$, which represents the linear response of a system to an external electromagnetic field. It can be expressed as $\varepsilon(\omega)=\varepsilon_{1}(\omega)+i \varepsilon_{2}(\omega)$, where $\varepsilon_{1}(\omega)$ and $\varepsilon_{2}(\omega)$ are the real and imaginary parts of the dielectric function, respectively. Generally, there are two contributions to $\varepsilon(\omega)$, namely, intra-band and inter-band transitions. The contribution from intraband transitions is crucial only for metals. The interband transitions can further be split into direct and indirect transitions. The indirect inter-band transitions which involved scattering of phonon was ignored. The imaginary part $\varepsilon_{2}(\omega)$ is directly related to the electronic band structure and it can be computed by summing up all possible transitions from the occupied to the unoccupied states, taking into account the appropriate transition dipole matrix elements. A full detail description of the calculation of these matrix elements is given by Ambrosch-Draxl et al. ${ }^{17}$. The real part $\varepsilon_{1}(\omega)$ can be derived from the imaginary part using the familiar Kramers-Kronig transformation. The knowledge of both real and imaginary parts of the dielectric function allows the calculation of various optical constants, such as the spectral reflectivity, the refractive index, and the electron energy loss function using standard expressions ${ }^{18}$.

Absorption coefficient is given according to TaucPankove relation ${ }^{19}$

$\alpha \mathrm{E}=\mathrm{B}\left(\mathrm{E}-\mathrm{E}_{\mathrm{g}}\right)^{\mathbf{p}}$

where $E$ is the photon energy, and $B$ is a factor depends on the transition probability and can be assumed to be constant within the optical frequency range, and the index $p$ is related to the distribution of the density of states. The index $p$ has discrete values like $1 / 2,3 / 2,2$, more depending on whether the transition is direct or indirect and allowed or forbidden. 


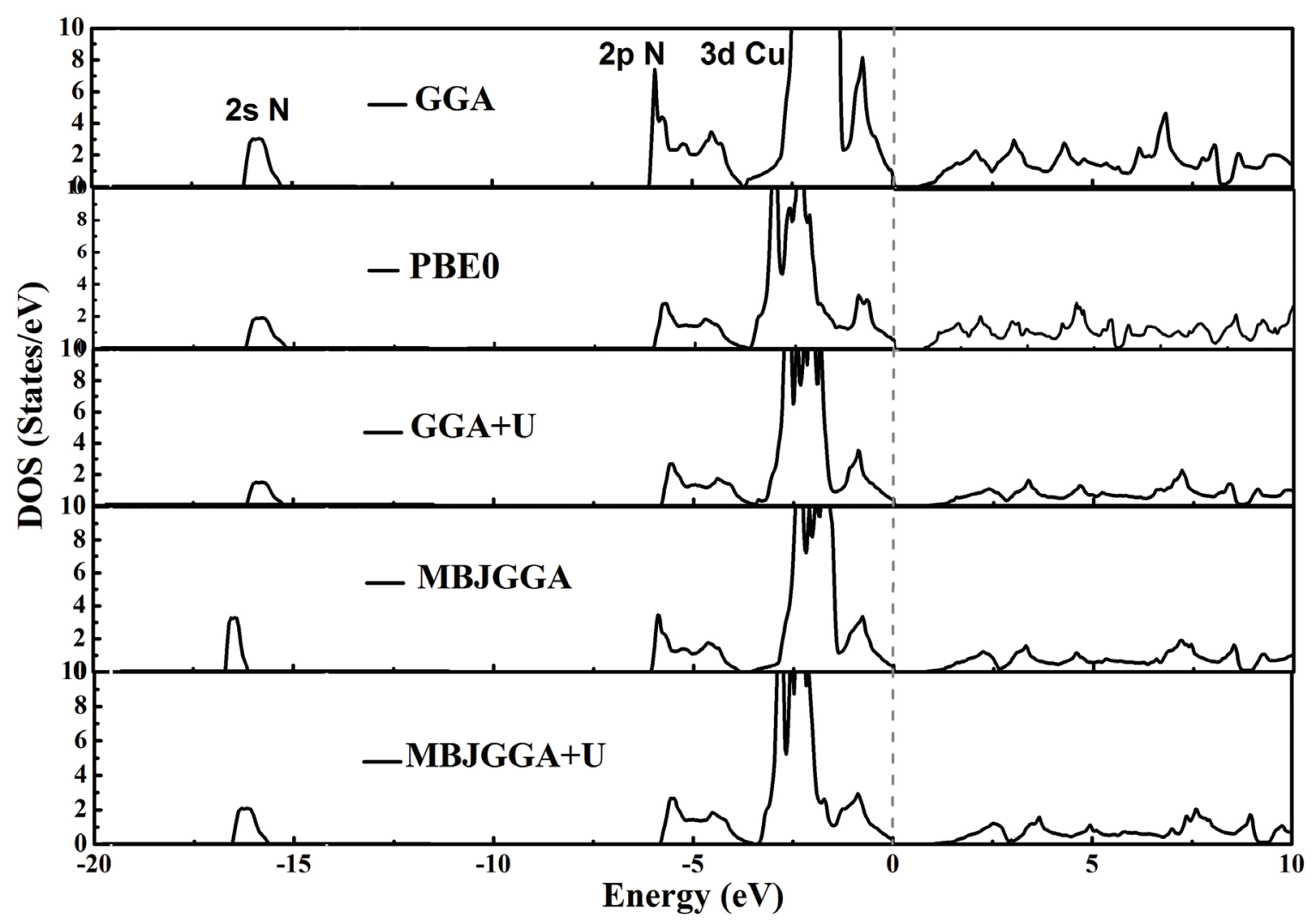

Figure 4. Total and partial density of states of $\mathrm{Cu}_{3} \mathrm{~N}$ at the ground state by using GGA, PBE0, GGA+U, MBJ and MBJ+U approximations.

Table 2. The calculated bandgap energy and comparison with other results.

\begin{tabular}{|c|c|c|c|c|c|c|c|}
\hline \multicolumn{2}{|c|}{ Other results } & \multirow{2}{*}{$\mathbf{M B J}+\mathbf{U}$} & \multirow{2}{*}{ MBJ } & \multirow{2}{*}{$\mathbf{G G A}+\mathbf{U}$} & \multirow{2}{*}{ PBEO } & \multirow{2}{*}{\multicolumn{2}{|c|}{ GGA }} \\
\hline Experimental $^{6,9,13}$ & Theoretical $^{1,7,8}$ & & & & & & \\
\hline $0.8-1.9$ & $0.23-0.9$ & 1.12 & 1.04 & 0.95 & 0.85 & 0.7 & Bandgap energy $(\mathrm{eV})$ \\
\hline
\end{tabular}

The optical energy gap was deduced from the intercept of the extrapolated liner part of the plot of $(\alpha E)^{1 / p}$ versus the photon energy $E$ with abscissa. The band energy $\mathrm{Cu}_{3} \mathrm{~N}$ is calculated according to $(\alpha E)^{1 / p_{-}}$E using Figure 5ain the frame of GGA, GGA+U and MBJ approaches. the optical band gap from these approaches were estimated $0.9,1.3$ and $1.5 \mathrm{eV}$, respectively.

The refractive index dispersion was calculated using GGA, GGA+U and MBJ approaches (Figure 5b). The phonon contributions are not included to the dielectric screening. $\varepsilon_{1}(\omega)$ corresponds to the static optical dielectric constant. The static refractive index was 3.3,2.9 and 2.7 for GGA, GGA+U and MBJ, respectively.

Transmittance and reflectance spectra were measured for as-deposited $\mathrm{Cu}_{3} \mathrm{~N}$ thin films by reactive magnetron sputtering at different substrate temperature ${ }^{9}$. Figure $6 a$ depicts the plot of Tauc- pankove relation versus photon energy. It is seen the theoretical and experimental bandgap energy are in good agreement. Figure $6 \mathrm{~b}$ illustrates the refractive index dispersion of the $\mathrm{Cu}_{3} \mathrm{~N}$ thin films. Cauchy dispersion relation ${ }^{20}$ $\mathrm{n}(\lambda)=\mathrm{n}_{0}+\mathrm{n}_{1} \mathrm{E}^{2}+\mathrm{n}_{2} \mathrm{E}^{4}$

was fitted with refractive index extracted from experimental transmittance and reflectance data and the $\mathrm{n}_{0}$, static refractive index, was obtained as 2.66-3.04 for $\mathrm{Cu}_{3} \mathrm{~N}$ thin films (see the corresponding theoretical value).

\subsection{Electronic charge distribution}

Figure 7 depicts the electronic charge distribution in (110) plane of $\mathrm{Cu}_{3} \mathrm{~N}$ structure. The electon cloud distribution slightly deformed between neighbors $\mathrm{Cu}-\mathrm{N}$ bond. To confirm the iconicity of the bound, according to HanneySmyth relation ${ }^{21}$, the iconicity of A- B bond is

$t i(\mathrm{HS})=0.16\left|\chi_{\mathrm{A}}-\chi_{\mathrm{B}}\right|+0.35\left|\chi_{\mathrm{A}}-\chi_{\mathrm{B}}\right|^{2}$

where $\chi_{\mathrm{A}}$ and $\chi_{\mathrm{B}}$ is the electronegativity of the involving atoms. $\chi_{\mathrm{Cu}}=1.90$ and $\chi_{\mathrm{N}}=3.04$. The iconicity of the chemical $\mathrm{Cu}-\mathrm{N}$ bound is around 0.63 . Hence, $\mathrm{Cu}_{3} \mathrm{~N}$ is mostly ionic. It confirms small deformation in electronic distribution between $\mathrm{Cu}-\mathrm{N}$ bound. 


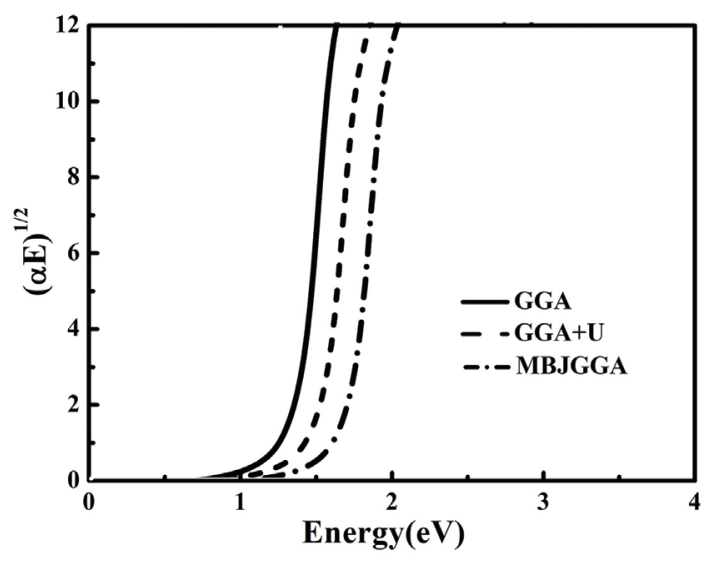

(a)

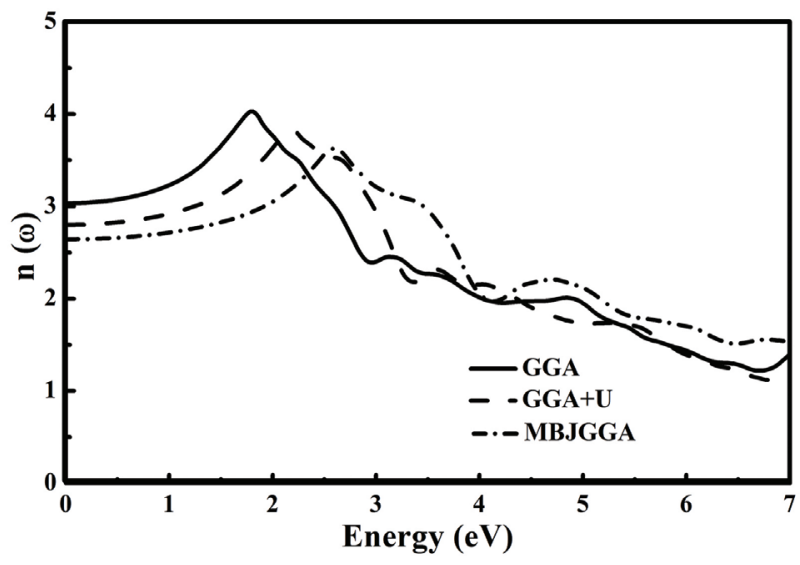

(b)

Figure 5. (a) The $(\alpha \mathrm{E})^{1 / 2}$-E to calculate the band gap energy (b) the refractive coefficient of $\mathrm{Cu}_{3} \mathrm{~N}$.

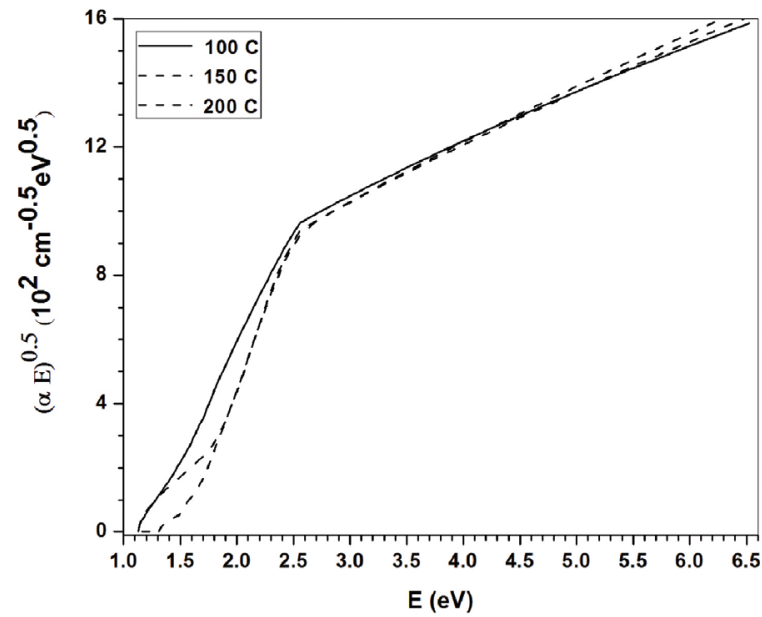

(a)

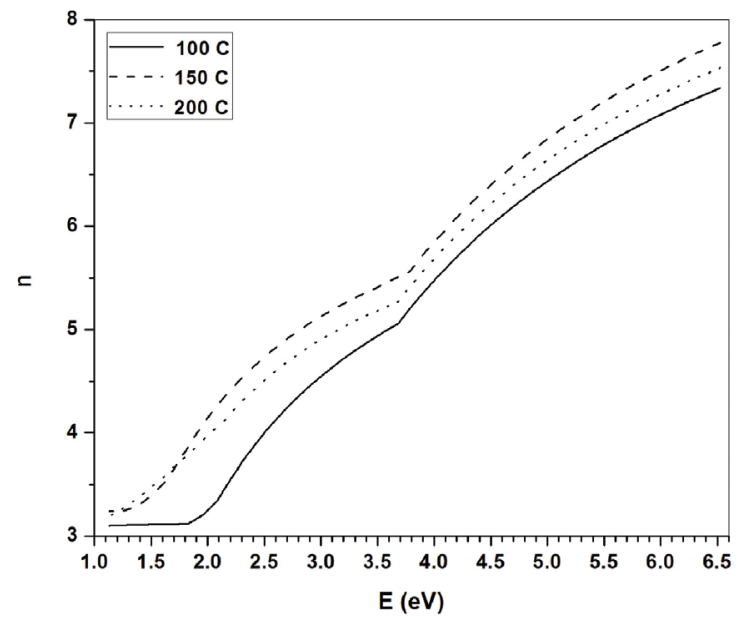

(b)

Figure 6. The plot of Tauc- Pankove relation (a) and dispersion of refractive index (b). (The related data are extracted from measured absorption coefficient, transmittance and reflectance).

\subsection{Elastic constants and mechanical properties}

The elastic constants $\mathrm{C}_{\mathrm{ij}}$ are the proportionality coefficients relating the applied strain $\left(\varepsilon_{\mathrm{i}}\right)$ to the stress $\left(\sigma_{\mathrm{i}}\right)$, $\sigma_{\mathrm{i}}=\mathrm{C}_{\mathrm{ij}} \varepsilon_{\mathrm{i}}$. So, $\mathrm{C}_{\mathrm{ij}}$ determines the response of the crystal to external forces ${ }^{22}$. Ab initio calculation of the elastic constants is based on induced changes in the total energy due to the changes in the strain ${ }^{23-25}$.

The symmetry of cubic crystal lattice reduces the 21 elastic constants to three independent elastic constants namely $\mathrm{C}_{11}, \mathrm{C}_{12}$ and $\mathrm{C}_{44}{ }^{[26]}$. The present values of elastic constants of $\mathrm{Cu}_{3} \mathrm{~N}$ using GGA were given in Table 3 . The elastic stability criteria for a cubic crystal $^{27}$ at ambient condition ( $\mathrm{P}=1 \mathrm{bar}$ and room temperature) are simultaneously $\mathrm{C}_{11}+2 \mathrm{C}_{12}>0, \mathrm{C}_{44}>0$ and $\mathrm{C}_{11}-\mathrm{C}_{12}>0$. The elastic constants were calculated using the tetrahedral and rhombohedral distortions on the cubic structure. The systems are fully relaxed after each distortion in order to reach the equilibrium $s_{a t e}{ }^{28}$. This optimization procedure was important for rhombohedral case, in which the accurate results could be obtained for $\mathrm{C}_{44}$. The elastic anisotropy ratio is another important physical quantity which tells about the structural stability and it is highly correlated with the possibility of inducing micro-cracks in the materials. This is defined as $\mathrm{A}=2 \mathrm{C}_{44} /\left(\mathrm{C}_{11}-\mathrm{C}_{12}\right)$. For a completely isotropic system, $\mathrm{A}$ is unity and the deviation from unity measures the degree of elastic anisotropy ${ }^{28}$. This factor was also calculated. The anisotropy factor values of $\mathrm{Cu}_{3} \mathrm{~N}$ do not deviate much from the unity, hence $\mathrm{Cu}_{3} \mathrm{~N}$ was not described by a profound anisotropy.

Voigt-Reuss ${ }^{29-30}$ approach, the actual effective modulus for crystals could be approximated by the arithmetic mean of the two well-known bounds for mono crystals. Then the main mechanical parameters for cubic structures, i.e. shear modulus G, bulk modulus B, Young's modulus E, Poisson's ratio $\sigma$ and Lame's coefficients $\mu$ and $\lambda$ were calculated from the elastic constants of the single crystals using the following relations:

$\mathrm{G}=\left(\mathrm{C}_{11}-\mathrm{C}_{12}+3 \mathrm{C}_{44}\right) / 5$ 
Table 3. The elastic constants of $\mathrm{Cu}_{3} \mathrm{~N}$.

\begin{tabular}{cccccccccccc}
\hline Elastic constants & $\mathbf{C}_{11}(\mathbf{G P a})$ & $\mathbf{C}_{\mathbf{1 2}}(\mathbf{G P a})$ & $\mathbf{C}_{\mathbf{4 4}}(\mathbf{G P a})$ & $\mathbf{B}(\mathbf{G P a})$ & $\mathbf{E}(\mathbf{G P a})$ & $\mathbf{A}$ & $\sigma$ & $\mu(\mathbf{G P a})$ & $\lambda(\mathbf{G P a})$ & $\mathbf{G}(\mathbf{G P a})$ & $\mathbf{B} / \mathbf{G}$ \\
\hline Peresent work & 249.910 & 49.557 & 35.090 & 116.342 & 156.045 & 0.35 & 0.28 & 60.94 & 77.58 & 61.125 & 1.9 \\
Others results $^{[22]}$ & 234.2 & 54.4 & 17.5 & 114.3 & 122.8 & - & - & - & & 46.5 & 2.4 \\
\hline
\end{tabular}

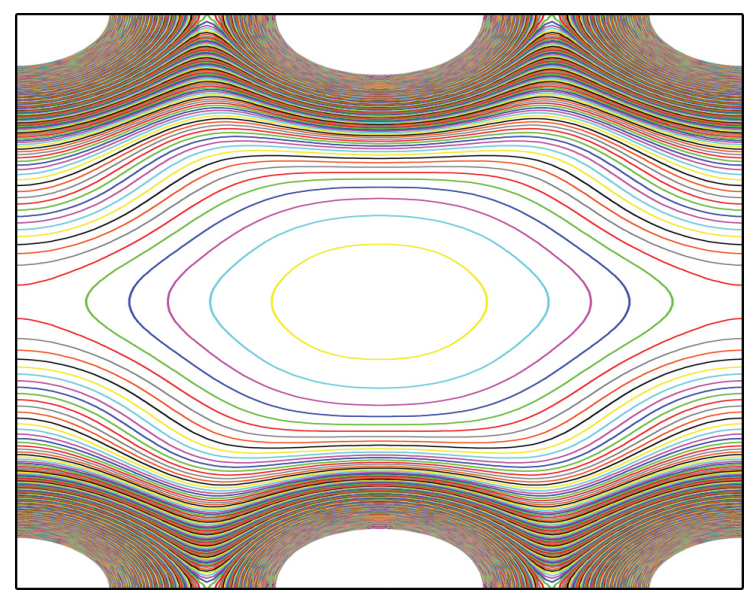

Figure 7. Electron density maps of $\mathrm{Cu}_{3} \mathrm{~N}$ in the (110) plane.

$\mathrm{B}=\frac{1}{3}\left(\mathrm{C}_{11}-2 \mathrm{C}_{12}\right)$

$E=9 B G /(3 B+G)$

$\sigma=(3 \mathrm{~B}-\mathrm{E}) /(6 \mathrm{~B})$

$\mu=\mathrm{E} / 2(1+\sigma)$

$\lambda=\sigma \mathrm{E} /\{(1+\sigma)(1-2 \sigma)\}$

The value of the Poisson ratio $\sigma$ for covalent materials is small as $\sigma=0.1$, whereas for ionic materials a typical value of $\sigma$ is $0.25^{[31]}$. The value of $\sigma$ was calculated to be about 0.28 , i.e. a ionic contribution in intra-atomic bonding for $\mathrm{Cu}_{3} \mathrm{~N}$ was confirmed (see section 3.4).

The bulk modulus B as calculated from elastic constant (116.342GPa) is in good agreement with the corresponding data in Table 1. According to the empirical Pugh formula ${ }^{32}$, the critical value of the ratio $\mathrm{B} / \mathrm{G}$ separates the ductile and brittle behavior of materials is around 1.75 ; i.e. if $\mathrm{B} / \mathrm{G}>$ 1.75 , the material behaves in a ductile manner; otherwise the material behaves in a brittle manner. Therefore, $\mathrm{Cu}_{3} \mathrm{~N}$ was classified as brittle material.

Using the Young's modulus E, bulk modulus B, and shear modulus $\mathrm{G}$, mean velocity $\left(\mathrm{v}_{\mathrm{m}}\right)$ and Debye temperature $\left(\theta_{D}\right)$ could been calculated. $\theta_{D}$ is an important fundamental parameter closely related to many physical properties such as elastic constants, specific heat and melting temperature. $\theta_{D}$ can be estimated from the elastic constants data, by the following classical relations ${ }^{33}$ :

$\theta_{D}=\frac{\mathrm{h}}{\mathrm{k}_{\mathrm{B}}}\left[\frac{3}{4 \pi V_{a}}\right]^{1 / 3} v_{m}$
Table 4. The calculated density $\left(\rho\right.$, in $\left.\mathrm{g} / \mathrm{cm}^{3}\right)$, longitudinal, transverse and average sound velocity $\left(\mathrm{v}_{\mathrm{l}}, \mathrm{v}_{\mathrm{t}}\right.$ and $\mathrm{v}_{\mathrm{m}}$, in $\left.\mathrm{m} / \mathrm{s}\right)$ and Debye temperature $\left(\theta_{\mathrm{D}}\right.$, in $\left.\mathrm{K}\right)$ for $\mathrm{Cu}_{3} \mathrm{~N}$.

\begin{tabular}{cccccc}
\hline Compound & $\rho$ & $\mathbf{v}_{\mathbf{t}}$ & $\mathbf{v}_{\mathbf{1}}$ & $\mathbf{v}_{\mathbf{m}}$ & $\theta_{\mathbf{D}}$ \\
\hline $\mathrm{Cu}_{3} \mathrm{~N}$ & 3.64 & 4097.9 & 7372.4 & 4563.9 & 353 \\
\hline
\end{tabular}

where $\mathrm{v}_{\mathrm{m}}$ is the mean sound velocity, $\mathrm{h}$ is Plank's constant, $\mathrm{k}_{\mathrm{B}}$ is Boltzmann's constant and $\mathrm{V}_{a}$ is the atomic volume. The mean sound velocity in the polycrystalline material is given by ${ }^{34}$ :

$v_{m}=\left[\frac{1}{3}\left(\frac{2}{v_{t}^{3}}+\frac{1}{v_{l}^{3}}\right)\right]^{-1 / 3}$

where $v_{1}$ and $v_{t}$ are the longitudinal and transverse elastic wave velocities, respectively, in an isotropic material, which can be obtained using the shear modulus $\mathrm{G}$ and the bulk modulus B from Navier's equation ${ }^{35}$ :

$v_{l}=\left[\frac{3 B+4 G}{3 \rho}\right)^{1 / 2}$ and $v_{t}=\left(\frac{G}{\rho}\right)^{1 / 2}$

The calculated sound velocity and Debye temperature as well as the density for $\mathrm{Cu}_{3} \mathrm{~N}$ are given in Table 4 . Unfortunately, as far as the authors know, there are no data available related to these properties in the literature for this compound. Future experimental work will testify the calculated results.

\subsection{The cohesive energy}

First principles calculations based on the DFT is a useful tool to determine the cohesive energy of the solids. The cohesive energy is defined as the difference between the energy of an isolated atom and the energy of the same atom in the solid. In order to calculate cohesive energy, the energy of an isolated atom by considering it to be in a large unit cell containing just one atom, with FCC crystal structure irrespective of crystal structure of the corresponding solid. This does not introduce any errors in the results, due to the fact that a very large unit cell has been considered in the calculations. The size of the unit cell was chosen sufficiently large so that the energy convergence with respect to the size of the cell was less than $0.0001 \mathrm{Ry}$ : a large cubic cell with dimensions of 25 a.u. was used for $\mathrm{Cu}$ and $\mathrm{N}$. The energy of isolated $\mathrm{Cu}$ and $\mathrm{N}$ atoms are $\mathrm{E}_{\text {tot }}(\mathrm{Cu})=-3309.77129537$ Ry and $E_{\text {tot }}(\mathrm{N})=-108.91055867 \mathrm{Ry}$, respectively. The energy of the same atom in the solid as $\mathrm{Cu}_{3} \mathrm{~N}$ is $\mathrm{E}_{\text {tot }}\left(\mathrm{Cu}_{3} \mathrm{~N}\right)=$ -10039.61508342Ry. The cohesive energy of $\mathrm{Cu}_{3} \mathrm{~N}$ is calculated as $\Delta E\left(C u_{3} N\right)=E_{\text {tot }}^{C u_{3} N}-\left(3 E_{\text {tot }}^{C u}-E_{\text {tot }}^{N}\right)=-1.39 R y$ 


\section{Conclusion}

In summary, ab-initio calculations of structural, electronic, optical and elastic properties of $\mathrm{Cu}_{3} \mathrm{~N}$ using full-potential linearized augmented plane-wave method. The calculations were done using different approaches for exchange-correlation term including GGA, LDA, PBE, and as well as new approximation MBJ-GGA. The addition of Hubbard potential parameter improved the predicted bandgap energy. The optimized structural parameters were in good agreement with the experimental ones. The elastic constants and derived bulk modulus, shear modulus, Young's modulus, Poisson's ratio, and sound velocities for longitudinal and shear waves were calculated.

The calculated elastic constants satisfied the stability criteria. The Debye temperature was calculated. The results showed that $\mathrm{Cu}_{3} \mathrm{~N}$ is brittle. Moreover, the results of electronegativity concept, electronic charge density and Poisson's ratio revealed that the bonding nature between $\mathrm{Cu}-\mathrm{N}$ was governed by ionic identity. The direct band

\section{References}

1. Moreno-Armenta MG, Soto G and Takeuchi N. Ab initio calculations of non-stoichiometric copper nitride, pure and with palladium. Journal of Alloys and Compounds. 2011; 509(5):1471-1476. http://dx.doi.org/10.1016/j. jallcom.2010.09.192

2. Borsa DM, Grachev S and Boerma DO. Development of epitaxial nitride-based bilayers for magnetic tunnel junctions. IEEE Transactions on Magnetics. 2002; 38(5):2709-2711. http://dx.doi.org/10.1109/TMAG.2002.803156

3. Asano M, Umeda $\mathrm{K}$ and Tasaki A. $\mathrm{Cu}_{3} \mathrm{~N}$ Thin Film for a New Light Recording Media. Japanese Journal of Applied Physics. 1990; 29:1985-1986. http://dx.doi.org/10.1143/JJAP.29.1985

4. Maruyama T and Morishita T. Copper nitride and tin nitride thin films for write once optical recording media. Applied Physics Letters. 1996; 69:890. http://dx.doi.org/10.1063/1.117978

5. Nosaka T, Yoshitake M, Okamoto A, Ogawa S and Nakayama Y. Thermal decomposition of copper nitride thin films and dots formation by electron beam writing. Applied Surface Science. 2001; 169-170:358-361. http://dx.doi.org/10.1016/ S0169-4332(00)00681-4

6. Navio C, Capitan M J, Alvarez J, Yndurain F and Miranda R. Intrinsic surface band bending in $\mathrm{Cu}_{3} \mathrm{~N}(100)$ ultrathin films. Physical Review B. 2007; 76:085105. http://dx.doi. org/10.1103/PhysRevB.76.085105

7. Armenta MGM, Martinez A and Takeuchi N. Ab initio total energy calculations of copper nitride: the effect of lattice parameters and $\mathrm{Cu}$ content in the electronic properties. Solid State Sciences. 2004; 6(1):9-14. http://dx.doi.org/10.1016/j. solidstatesciences.2003.10.014

8. Hou ZF. Effects of $\mathrm{Cu}, \mathrm{N}$, and $\mathrm{Li}$ intercalation on the structural stability and electronic structure of cubic $\mathrm{Cu}_{3} \mathrm{~N}$. Solid State Sciences. 2008; 10(11):1651-1657. http://dx.doi.org/10.1016/j. solidstatesciences.2008.02.013

9. Hadian F, Rahmati A, Movla H and Khaksar M. Reactive DC magnetron sputter deposited copper nitride nano-crystalline thin films: Growth and characterization. Vacuum. 2012; 86(8):10671072. http://dx.doi.org/10.1016/j.vacuum.2011.09.001 gap energy (about $1.12 \mathrm{eV}$ ) which obtained by $\mathrm{MBJ}+\mathrm{U}$, are well in agreement with experimental result. The static refractive index was 2.7-3.3 that extracted from dispersion of refractive index. The optical band gap energy obtained using Tauc-Ponkove relation is greater than that of predicted by band structure.

The static equilibrium lattice structure, elastic constants bulk modulus and Debye temperature of the cubic anti- $\mathrm{ReO}_{3}$ structural copper nitride $\left(\mathrm{Cu}_{3} \mathrm{~N}\right)$ were calculated using a firstprinciples full-potential linearized augmented plane wave (FP-LAPW). For the exchange-correlation terms, GGA, GGA + U, PBE0, MBJ and MBJ + U approximations have been used. The bandgap energy of $\mathrm{Cu}_{3} \mathrm{~N}$ has been calculated using effective potential U estimated by ab-initio study and improved with respect to the other theoretical works. The refractive index and optical gap have been calculated and compared with other studies. Moreover, mechanical stability of $\mathrm{Cu}_{3} \mathrm{~N}$ was confirmed using the calculated mechanical and elastic constants.

10. Gonzalez-Arrabal R, Gordillo N, Martin Gonzalez MS, Ruiz Bustos R and Agullo-Lopez F. Thermal stability of copper nitride thin films: The role of nitrogen migration. Journal of Applied Physics. 2010; 107:103513. http://dx.doi. org/10.1063/1.3369450

11. Yu W, Li LY and Jin CQ. Pressure induced metallization of $\mathrm{Cu}_{3}$ N. Journal of Materials Science. 2005; 40(17):4661-4664. http://dx.doi.org/10.1007/s10853-005-0638-3

12. Blaha KSP, Madsen G, Kvasnika D and Luitz K. WIEN2k, an Augmented Plane Wave Plus Local Orbitals Program for Calculating Crystal Properties. Wien: Karlheinz Schwarz, Techn. Universit€at Wien; 2001.

13. Monkhorst HJ and Pack JD. Special points for Brillouin-zone integrations. Physical Review B. 1976; 13(12):5188-5192. http://dx.doi.org/10.1103/PhysRevB.13.5188

14. Murnaghan FD. The compressibility of media under extreme pressures. Proceedings of the National Academy of Sciences of the United States of America. 1944; 30(9):244. http://dx.doi. org/10.1073/pnas.30.9.244

15. Georg K, Madsen $\mathrm{H}$ and Novak P. Calculation the effective $U$ in APW methods. NiO. 2007. Available from: <http://www. wien2k.at/reg_user/textbooks/Constraint_U.pdf>.

16. Tran F. and Blaha P. Accurate Band Gaps of Semiconductors and Insulators with a Semilocal Exchange-Correlation Potential. Physical Review Letters. 2009; 102:226401. http:// dx.doi.org/10.1103/PhysRevLett.102.226401

17. Ambrosch-Draxl C and Sofo JO. Linear optical properties of solids within the full-potential linearized augmented planewave method. Computer Physics Communications. 2006; 175(1):114. http://dx.doi.org/10.1016/j.cpc.2006.03.005

18. Delin A, Eriksson AO, Ahuja R, Johansson B, Brooks MSS, Gasche T et al. Optical properties of the group-IVB refractory metal compounds. Physical Review B. 1996; 54(3):1673-1681. http://dx.doi.org/10.1103/PhysRevB.54.1673

19. Patterson J and Bailey B. Solid-State Physics, Introduction to the theory. 2nd ed. Springer; 2010. 
20. Singh J. Optical Properties of Condensed Matter and Applications. John Willey \& Sons Ltd.; 2006. http://dx.doi. org/10.1002/0470021942

21. Hanney NB and Smyth CP. The Dipole Moment of Hydrogen Fluoride and the Ionic Character of Bonds. Journal of the American Chemical Society. 1946; 68(2):171-173. http:// dx.doi.org/10.1021/ja01206a003

22. Seddik T, Khenata R, Bouhemadou A, Reshak AH, Semari F and Amrani B. Prediction study of the structural, elastic and high pressure properties of Yttrium chalcogenide. Computational Materials Science. 2010; 49(2):372-377. http:// dx.doi.org/10.1016/j.commatsci.2010.05.024

23. Mehl MJ. Pressure dependence of the elastic moduli in aluminum-rich A1-Li compounds. Physical Review B. 1993; 47(5):2493-2500. http://dx.doi.org/10.1103/ PhysRevB.47.2493

24. Khenata R, Bouhemadou A, Reshak AH, Ahmed R, Bouhafs B, Rached D et al. First-principles calculations of the elastic, electronic, and optical properties of the filled skutterudites $\mathrm{CeFe}_{4} \mathrm{P}_{12}$ and $\mathrm{ThFe}_{4} \mathrm{P}_{12}$. Physical Review B. 2007; 75(19):195131. http://dx.doi.org/10.1103/PhysRevB.75.195131

25. Bouhemadou A, Khenata R, Zegrar F, Sahnoun M, Baltache $\mathrm{H}$ and Reshak $\mathrm{AH}$. Ab initio study of structural, electronic, elastic and high pressure properties of barium chalcogenides. Computational Materials Science. 2006; 38(2):263-270. http:// dx.doi.org/10.1016/j.commatsci.2006.03.001

26. Kittel C. Introduction to solid state physics. 8th ed. John Wiley \& Sons Inc.; 2005.
27. Born M and Hang K. Dynamical Theory and Experiments I. Berlin: Springer Verlag Publishers; 1982.

28. Voight W. Lehrbuch Der Kristallphysik. Johnson Reprint Corp; 1928.

29. Reuss A and Angew Z. Berechnung der fliessgrenze von mischkristallen auf grund der plastizitasbendingung fur einkristalle. Journal of Applied Mathematics and Mechanics. 1929; 9(1):40-58.

30. Edward S, Anderson OL and Schreiber SN. Elastic Constants and Their Measurement. New York: McGraw-Hill Education; 1974.

31. Haines J, Leger JM and Bocquillon G. Synthesis and design of superhard materials. Annual Review of Materials Research. 2001;31:1-23. http://dx.doi.org/10.1146/annurev.matsci.31.1.1

32. Pugh SF. Relation between the elastic moduli and the plastic properties of polycrystalline pure metals. Philosophical Magazine. 1954; 45:823-843

33. Wachter P, Filzmoser M and Rebizant J. Electronic and elastic properties of the light actinide tellurides. Physica B: Condensed Matter. 2001; 293(3-4):199-223. http://dx.doi.org/10.1016/ S0921-4526(00)00575-5

34. Anderson OL. A simplified method for calculating the debye temperature from elastic constants. Journal of Physics and Chemistry of Solids. 1963; 24(7):909-917. http://dx.doi. org/10.1016/0022-3697(63)90067-2

35. Schreiber E, Anderson OL and Soga N. Elastic Constants and their Measurements. New York: McGraw-Hill; 1996. 